\title{
Histological Description of Meningeal and Periosteal Dural Layers at the Porus of Internal Acoustic Canal in the Vestibular Schwannoma
}

\author{
Agung Budi Sutiono, ${ }^{1}$ M. Zafrullah Arifin, ${ }^{1}$ Ahmad Faried, ${ }^{1}$ Takayuki Ohira ${ }^{2}$ \\ ${ }^{1}$ Department of Neurosurgery, Faculty of Medicine, Universitas Padjadjaran-Dr. Hasan Sadikin General \\ Hospital \\ ${ }^{2}$ Department of Neurosurgery, Keio University School of Medicine, Tokyo, Japan
}

\begin{abstract}
Objective: To study the transformation point of meningeal and periosteal dural at the porus of internal acoustic canal (IAC) in order to verify the different thickness of meningeal and periosteal dura in vestibular schwannomas (VS).

Methods: Three IAC cadaver specimens and ten samples of VS patients from porus were obtained and analyzed. Samples were stained by using Masson trichrome technique after cutting in 6 micron of thickness. The samples were then observed under light microscopes to understand the meninges pattern in the IAC.

Results: The meningeal dura is becoming thin at the porus and disappears at the meatal portion to form epineurium. However, the periosteal dura is lining continuously to the fundus. In VS, the meningeal dura becomes thick and forms a pseudo-capsule in the middle of meatus, known as perineurium. The residual nerve filament was compressed by the tumor parenchyma. Between the tumor and nerve interface, three or more perineureal layers are seen. The perineurium in the cisternal portion was consistently loose and forms the tumor and arachnoid nerve interface. Almost all the nerve filaments are displaced to the tumor periphery near the pseudocapsule. In contrast, the periosteal dural of VS is becoming thin and disappear nearby the middle of meatal portion. This changing site establishes "meningo-periosteal ring" of VS because of the encircling nearby the porus.
\end{abstract}

Received:

November 28, 2012

Conclusions: In IAC, the meningeal dural becomes thin. The periosteal dura is lining continuously to the fundus. In VS, the meningeal dura becomes thick, joins perineurium and forms pesudocapsule near the porus, but the periosteal dura disappeared. This changing point is called meningo-periosteal ring.

Revised:

April 14, 2013

Accepted:

January 31, 2013

Keywords: Meningeal, periosteal, porus, vestibular schwannomas

IJIHS. 2013;1(1):22-8

\section{Introduction}

The concept from Yasargil et al. ${ }^{1,2}$ concerning the origin of vestibular schwannomas (VS) came from the outside of subarachnoid space. It is stated the growth of VS pushes the arachnoid wall to the cerebellopontine cistern medially and duplicates the arachnoid layers. In addition, the anatomical study in IAC revealed that the

\footnotetext{
Correspondence:

Agung Budi Sutiono, Department of Neurosurgery, Faculty of Medicine, Universitas Padjdajaran-Dr. Hasan Sadikin General Hospital

Jl. Pasteur No. 38 Bandung

e-mail: agungbudis@gmail.com
}

dura mater and the arachnoid invaginates into the IAC from porus to fundus, creating a lateral extension of cerebellopontine cistern, called acousticofacial cistern. VS originates from the vestibulocochleofacial complex which was contained in the acousticofacial cistern. ${ }^{3} \mathrm{~A}$ concept stated that the tumor capsule in the VS was actually formed by the residual vestibular nerve tissue itself, consisting of the perineurium and the underlying nerve fibers was established. ${ }^{4}$ However, the relation between the meningeal and periosteal dural structures and perineurium in the VS at the porus entrance site has not been entirely discussed. Therefore, this study aims to describe the relation among them. 


\section{Methods}

Ethical approval was obtained from the Ethical Committee of Keio University Hospital. Three formalin fixed adult cadaver head specimens were used and stained with Masson trichrome to observe the meninges structures in IAM (Fig. 1). Ten consecutive VS patients, operated by lateral suboccipital transmeatal approach, enrolled in this study after providing informed consent. Two samples from meatal and cisternal portions near the porus were obtained from one patient and other eight samples near the porus entrance were obtained from the patients. The sample location and characteristics of VS patients are presented (Table 1). The surgical specimens were fixed in formaldehyde, cut perpendicularly to the tumor surface, then embedded in paraffin, and sectioned at 4 micron of thickness. Stainings with hematoxylin eosin, Masson trichrome and the immunohistochemical myelin basic protein (MBP) were performed with Myelin basic protein staining performed using an enhanced, indirect immunoperoxidase technique, and the sections were lightly counterstained with hematoxylin. The primary antibody used was rabbit polyclonal anti-human MBP antibody. ${ }^{5}$

\section{Results}

Meninges pattern in IAM

Axial histological sections of the right IAC were viewed and stained with Masson trichrome. The two layers of dura mater, with the meningeal dura facing the subdural space and periosteal dura facing the bone, enter the IAM (Fig. 1) with the arachnoid invaginates into it. This leads to a creation of a dural cul de sac muff ${ }^{3,6}$ and this becomes thin in the fundus (Fig. 2a). However, the meningeal dura itself becomes thin and disappears at the middle of meatus (Fig. 2b) and the arachnoid transforms into the perineurium (Fig. 2c). Meanwhile, the periosteal dura drapes and follows the bone to the extracranial portion and becomes periosteum. The fasciovestibulo cochlear complex is wrapped with the arachnoid from porus and enters the subarachnoid space at the fundus level (Fig. 2a). The meningeal dura transforms into epineurium and the arachnoid transform into the perineurium. Meanwhile the endoneurium covers the nerve filaments (Fig. $2 d, e)$.

\section{Meninges pattern in the VS}

Histological sections of VS including the dura mater at the porus of IAC was stained with Masson trichrome. The thick meningeal dura follows the tumor and the thin periosteal dura lines on the bone surface and disappears after passing the porus. It also joins the arachnoid and perineurium, which covers and directly interfaces with the tumor (Fig. 3a). The periosteal dura completely disappears near the porus inside the IAC wall with only meningeal dura left and it is connected to the perineurium (Fig. 3b). At the level of transformation point from meningeal dura to the perineurium adjacent to the porus, the perineurium interfaces the tumor and also nerve en bloc (Fig. 3c). The histological section which was stained with hematoxylin eosin shows a changing point of meningeal and periosteal

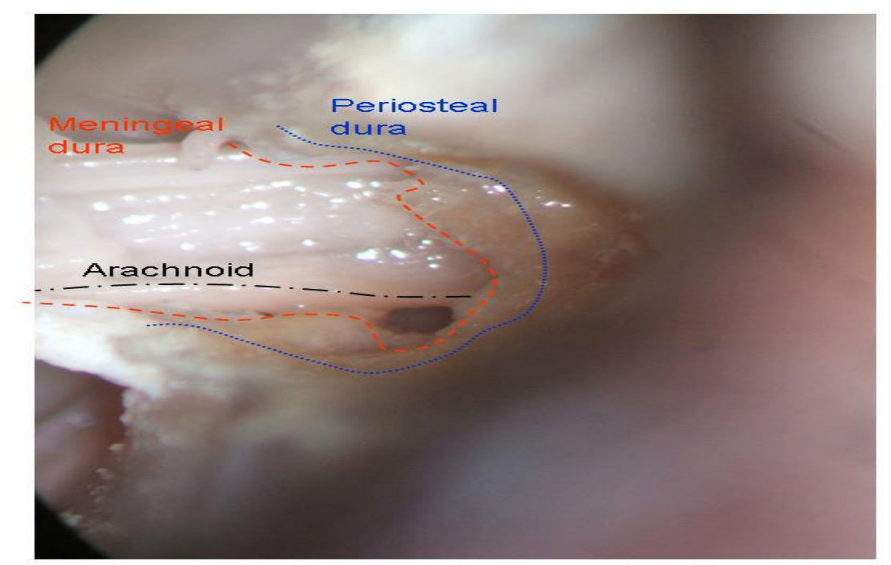

Fig. 1 The Right IAC from Cadaver Specimens. The posterior wall has been drilled out to expose the meatal portion. The meningeal and periosteal dural line the IAC, the arachnoid invaginates into the fundus and wraps the fasciocochleovestibular nerves complex 


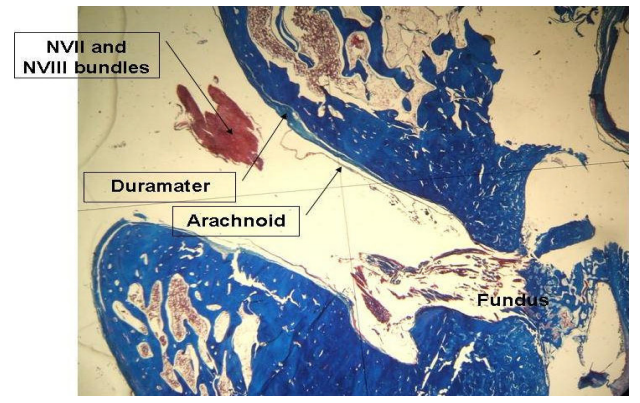

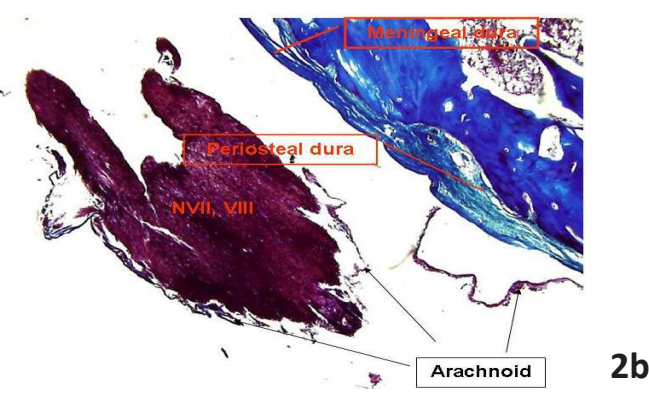

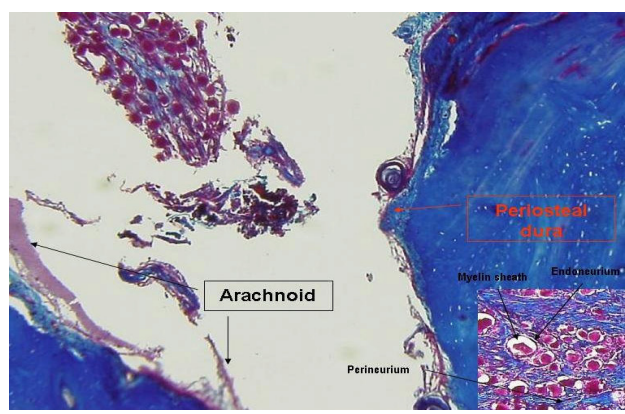

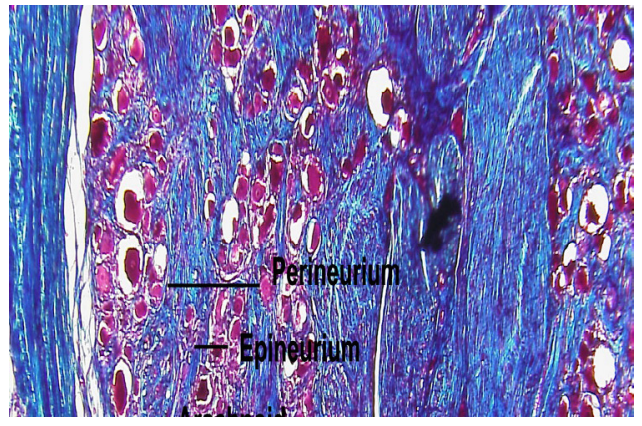

$2 e$

Fig. 2 The IAC Right Axial Section. The VII and VIII nerve complexes course and enter the porus. The dura mater lining is found on the bone IAC wall and the arachnoid invaginates from cistern of cerebelopontine angele towards the fundus making dural cul de sac (Fig. 2b). Fig. $2 a$ is an enlarged version at the cranial VII and VIII nerve complexes on which the meningeal dura becomes thin and periosteal dura continues towards the fundus level. The arachnoid wraps the nerves (Fig. 2C). Fig. $2 \mathrm{a}$ is an enlarged version at the fundus level, towards the facial canal. The arachnoid wraps the nerves and transforms into epineurium (Fig. 2 d). Fig. $2 a$ is an enlarged version at the fundus level towards the vestibular nerve. The periosteal dura becomes thin, arachnoid wraps the nerves and transforms into perineurium. Enlarging Fig. $2 \mathrm{~d}$ on the right below, the endoneurium is seen at the outer layer of myelin sheat (Fig. 2e). Fig. $2 \mathrm{c}$ is the enlarged version. Periosteal dura, meningeal dura, arachnoid, epineurium, perineurium stratification are seen from outside to the inside nerve filament bundle 
dura at the level of porus from the cisternal to the meatal portion. Periosteal dura becomes thin, meanwhile the meningeal dura is thicker than periosteal dura and transforms into perineurium (Fig. 3d). The Masson trichrome staining in the cisternal portion close to the porus shows that the perineurium forms pseudocapsule and is located between the arachnoid and the tumor, creating an arachnoid-tumor interface (Fig. 3e). The immunohistochemical myeline basic protein reveals the nerve filaments located on the pseudocapsule near the arachnoid due to the compression of the tumor parenchyma (Fig. 3f).

\section{Discussion}

The fate of meningeal-periosteal dura pattern in VS has not given considerable discussions in the past. This study shows that the pattern of meninges has different dura mater characteristics in the porus IAC with VS compared to the normal anatomy. The meningeal dura joins perineurium and intermingles gradually, tightly adheres with the tumor (Fig. 3a, b, c). It was considered that the pseudocapsule formation starts near the porus of IAC wall but it is less prominent. Periosteal dura becomes thin and disappears after passing through the porus in the meatal portion (Fig. 3d). Tumor beyond the meatus is covered by perineurium and arachnoid leading to pseudocapsule development (Fig. 3e). The application of immunohistochemical myelin basic protein staining shows that the residual vestibular nerve filament is compressed by the Schwann cell tumor. The residual nerve filaments are displaced near the tumor capsule (Fig. 3f).

Concurrently, the cadaver specimen staining shows normal strucutures of IAC axial section (Fig. 1). The dura mater consists of periosteal and meningeal dura which cover the bonny wall of IAM at the porus level. The periosteal dura gradually becomes thin towards the fundus, but the meningeal dura disappears at the middle part of IAC (Fig. 2a, b). The periosteal dura remains and continues lining into the fundus level to the extracranial portion to become periosteum (2c, d). The meningeal dura joins perineurium that is transformed from arachnoid (Fig. 2e).

Based on these findings, different patterns of dura mater are seen in the normal IAC and VS sections. In the normal cadaver specimen section, the periosteal dura lines the bone surface inside the meatus but the meningeal dura disappears. Conversely, in the VS specimen the periosteal dura disappears after passing the porus. However, the meningeal dura transforms into epineurium and the arachnoid becomes perineurium. Both meninges pattern forms a meningoperiosteal dura circle that lines the meatal wall to create a "meningoperiosteal ring" (Fig. 4a, b). The meningoperiosteal ring is an overlying two types of dura mater. It is located between meningeal dura and periosteal dura in which the periosteal dura faces the bone and disappears. The meningeal dura connects to the

Table 1 Characteristic Patients with Vestibular Schwannoma

\begin{tabular}{|c|c|c|c|c|}
\hline No. & $\begin{array}{l}\text { Gender/Age } \\
\text { (years) }\end{array}$ & Size $(\mathrm{cm})$ & Symptoms & Sample location \\
\hline 1 & $F, 45$ & 3.7 & Tinnitus and hearing disturbance & $\begin{array}{l}\text { Inside and outside meatus near porus } \\
\text { separately }\end{array}$ \\
\hline 2 & $\mathrm{M}, 30$ & 1.4 & Tinnitus and hearing disturbance & Porus inside meatus \\
\hline 3 & M, 58 & 2.4 & Hearing disturbance & $\begin{array}{l}\text { Inferior vestibular nerve en bloc near } \\
\text { porus inside meatus }\end{array}$ \\
\hline 4 & $F, 31$ & 1.2 & Hearing loss & $\begin{array}{l}\text { Inside meatus near porus including } \\
\text { duramater }\end{array}$ \\
\hline 5 & $F, 62$ & 3.2 & Hearing loss and dizziness & $\begin{array}{l}\text { Inside meatus near porus including } \\
\text { arachnoid and duramater }\end{array}$ \\
\hline 6 & $F, 62$ & 2.3 & Hearing loss & Outside meatus near porus \\
\hline 7 & $M, 69$ & 4.8 & Hearing disturbance & Cystic outside meatus near porus \\
\hline 8 & $M, 54$ & 1.7 & Tinnitus & Porus entrance including duramater \\
\hline 9 & $F, 41$ & 2.6 & Tinnitus & Porus entrance including duramater \\
\hline
\end{tabular}



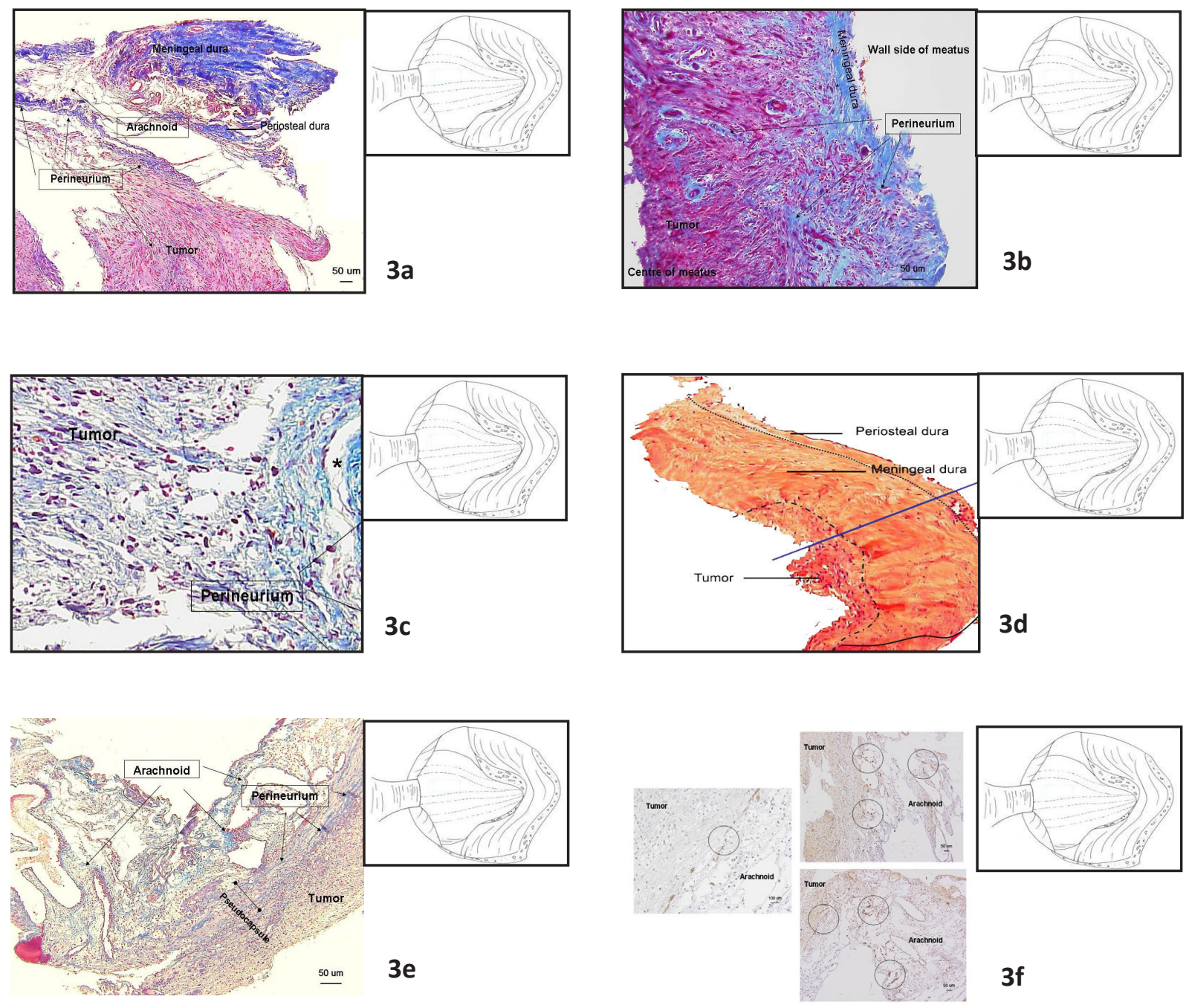

Fig. 3 VS Sample Stained with Masson Trichrome was Taken Near Porus as Shown on the Scheme Figure on the Right Upper. The meningeal dura and periosteal dura is folding due to the staining process. The meningeal dura is thicker than periosteal dura, then periosteal dura disappears. The arachnoid transforms into perineurium, faces the tumor and connects to the meningeal dura (Fig. 3b). The sample is taken from meatus, nearby the fundus, from the meatal wall. The epineurium is interconnecting with meningeal dura. It might be the beginning of pseudocapsule but less prominent. Note that the periosteal dura is not observed on this section, although the sample is taken from the dura which attaches on the meatal bone (Fig. 3c). Sample was taken from meatus nearby porus. The meningeal dura connects to the perineurium. The perineurium borders the nerve and tumor interface (Fig. 3d).Sample stained with Hematoxylin eosin was taken from cistern to the meatus portion, passing through the porus. The periosteal dura which faces the bone becomes thin and disappears after passing the porus. The thick meningeal dura remains and transforms into perineurium in the meatal portion. The tumor interfaces the perineurium forming a pseudocapsule in the cisternal portion (Fig. 3e). Sample was taken from meatal portion. The perineurium interfaces the tumor and arachnoid, creating a pseudocapsule (Fig. 3f). Sample from the meatal portion was stained with myelin basic protein to observe nerve filament. The residual nerve filaments exist on the tumor surface near the arachnoid 
$4 a$

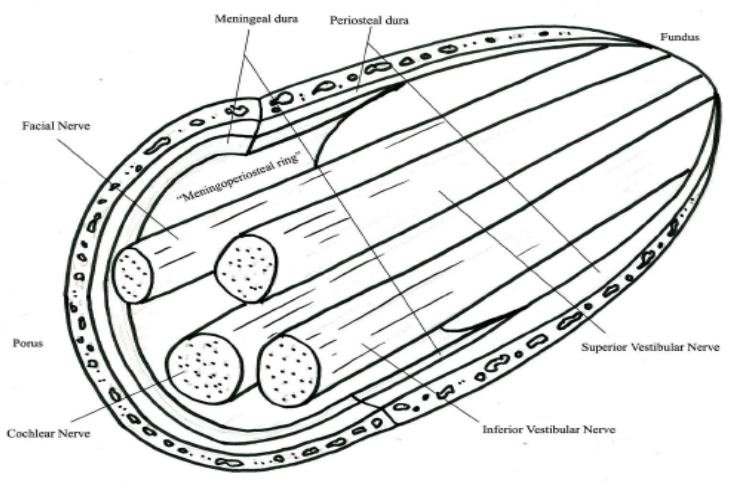

4b

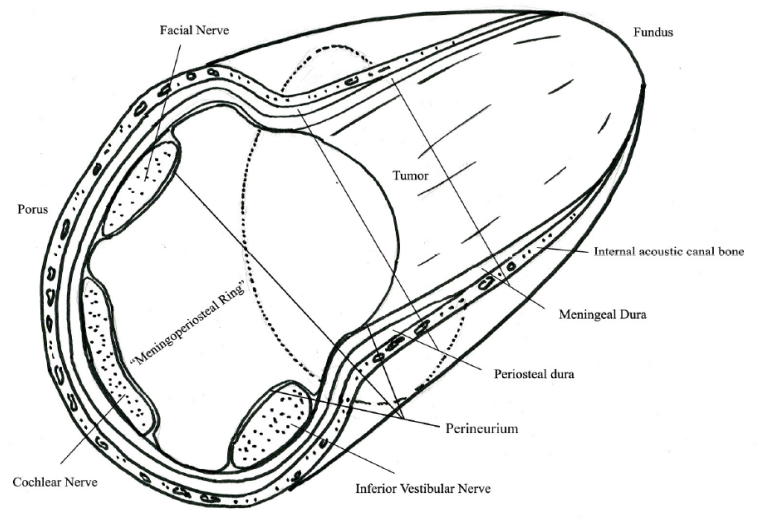

Fig. 4 Conceptual Scheme of the Meningeal Dura and Periosteal Dura. In the normal structure of IAC, the periosteal dura lines the bone of the meatal wall from porus toward fundus continuously, become periosteum to the extracranial part. The meningeal dura which faces the meatal cistern becomes thin near the middle of meatus and disappears. From this point a "meningoperiosteal ring" begins towards the porus (Fig. 4b). The scheme of VS shows that the periosteal dura that lines the bone becomes thin and disappears near the middle of the meatal portion, and this point is the beginning of the "meningoperiosteal ring" towards the porus. The meningeal dura remains and joins with the perineurium, interfacing the tumor and likely to be the beginning of pseudocapsule

perineurium to form the pseudocapsule. The meningoperiosteal ring can be found on the meatal, porus, or cisternal portions due to the widening and compression of the tumor (Table 2). However, it needs further investigations.

In the process of the tumor enlargement, the tumor compresses and displaces the perineurium from the origin of tumor nerve filament towards the meatal wall to develop a perineural pattern with different thickness. The perineurium thickness variations before it transforms into meninges could be related to the topographical anastomoses between facial and vestibular nerves. The anastomoses between facial and vestibular nerves are found in $83 \%$, of which $67 \%$ of them are near porus and $33 \%$ are between intermedial and lateral portions of IAM. The other variation is also found regarding the position of facial nerve towards the vestibulochoclear nerve, of which the anterioinferior to the vestibulochoclear nerve is more dominant than the anterosuperior. ${ }^{7-11}$

Table 2 Comparison of Meningeal and Periosteal Dural Pattern of in VS and Cadaver Specimens

\begin{tabular}{|c|c|c|c|}
\hline No. & Meninges & In vestibular schwannoma & In cadaver specimens \\
\hline 1 & Periosteal dura & $\begin{array}{l}\text { Line up to medial part of meatus and } \\
\text { disappear }\end{array}$ & $\begin{array}{l}\text { Line to the fundus and continue to the } \\
\text { periosteum }\end{array}$ \\
\hline 2 & Meningeal dura & $\begin{array}{l}\text { Attach to the tumor surface and } \\
\text { transform into perineurium }\end{array}$ & $\begin{array}{l}\text { Line up to medial part of meatus and } \\
\text { disappear }\end{array}$ \\
\hline 3 & Arachnoid & Line up to porus entrance & $\begin{array}{l}\text { Line to the fundus making } \\
\text { invagination cul de suc a muf }\end{array}$ \\
\hline 4 & $\begin{array}{l}\text { "Meningoperiosteal } \\
\text { ring" }\end{array}$ & $\begin{array}{l}\text { Begin from the end of periosteal } \\
\text { dura towards porus, meningeal dura } \\
\text { remains and joins the perineurium, } \\
\text { a likely to be the beginning of the } \\
\text { pesudocapsule }\end{array}$ & $\begin{array}{l}\text { Begin from the end of meningeal } \\
\text { dura towards porus, periosteal dura } \\
\text { remains continuous to become } \\
\text { periosteum to the extracranial portion }\end{array}$ \\
\hline
\end{tabular}


In conclusion, in the IAC, the meningeal dural becomes thin and disappears. The periosteal dural lines continuously and follows the bone to the fundus to form the periosteum. Conversely, in VS, the meningeal dura becomes thick and transforms into perineurium to form pesudocapsule in the middle part of meatus towards porus entrance; but in this part, the periosteal dura disappears. This changing point

\section{References}

1. Yasargil M, Smith R, Gasser J. Microsurgical approach to acoustic neurinomas. In: Krayenbuhl $\mathrm{H}$, editor. Advanced and technical standards in neurosurgery. Vol. 4. Vienna, Austria: SpringerVerlag; 1977. p. 93-129

2. Yasargil MG, Kasdaglis K, Jain KK, Weber HP. Anatomical observations of the subarachnoid cisterns of the brain during surgery. J Neurosurg. 1976;44:298-302.

3. Lescanne $E$, Velut $S$, Lefranco $T$, Destrieux $C$. The internal acoustic meatus and its meningeal layers a microanatomical study. I Neursurg. 2002;97:1191-97.

4. Sasaki T, Shono T, Hashiguchi K, Yoshida F, Suzuki SO. Histological considerations of the cleavage plane for preservation of facial and cochlear nerve functions on vestibular schwannoma surgery. J Neurosurg. 2009 Apr;110(4):648-55.

5. Keita M, Magy L, Richard L, Piaser M, Vallat J. LR white post-embedding colloidal gold method to immunostain MBP, PO, NF and S100 in glutaraldehyde fixed peripheral nerve tissue. J Peripheral Nerv Syst. 2002;7:128-33. is called the "meningoperiosteal ring".

Acknowledgement

We would like to thank to Prof. Sadakazu Aiso and Dr. Nobuaki Imanishi from Department of Clinical Anatomy Keio University for the kind support during this study and also to Mrs. Kiyomi Koide and Mrs. Naoko Tsuzaki for their kind assistance during the histological staining.

6. Lescanne E, Francois P, Velut S. Cerebellopontice cistern: microanatomy applied to the vestibular schwannomas. Prog Neurol Surg. 2008;21:43-53.

7. Tian G, Da chuan Xu, Huang D, Liao H, Huang M. The topographical relationship and anastomoses of the nerves in the human internal auditory meatus. Surg Radiol Ant. 2008;30:234-47.

8. Kuo TC, Jackler RK, Wong K, Blevins NH, Pitts LH. Are acoustic neuromas encapsulated tumors? Otolaryngol Head Neck Surg. 1997;117:606-9.

9. Ohata K, Tsuyuguchi N, Morino M, Takami T, Goto T, Hakuba A, et al. A hypothesis of epiarachnoidal growth of vestibular schwannoma at the cerebello-pontine angle: surgical importance. J Postgrad Med. 2002;48:253-9.

10. Thomas PK. Change in the endoneural sheaths of peripheral myelinated nerve fibers during wallerian degeneration. J Anat. 1964;98(2):17582.

11. Lihua $C$, Ling $C$, LiXu L, Feng L, Xianrui Y, Jiasheng F, et al. Vestibular schwannoma microsurgery with special reference to facial nerve preservation. Clin Neurol Neurosurg. 2009;111:47-53. 